\title{
Parallel-Stacked Flexible Organic Light-Emitting Diodes for Wearable Photodynamic Therapeutics and Color-Tunable Optoelectronics
}

Yongmin Jeon ${ }^{1}$, Ilkoo Noh'2, Young Cheol Seo ${ }^{1}$, Jun Hee Han ${ }^{1}$, Yongjin Park ${ }^{1}$, Eun Hae Cho ${ }^{1}$ and Kyung Cheol Choi ${ }^{* *}$

${ }^{1}$ School of Electrical Engineering, Korea Advanced Institute of Science and Technology (KAIST), Daejeon 34141, Republic of Korea.

2Department of Biological Science, Korea Advanced Institute of Science and Technology (KAIST), Daejeon 34141, Republic of Korea.

"Corresponding author: kyungcc@kaist.ac.kr (Kyung Cheol Choi) 


\section{Supplementary Figures}

(a)
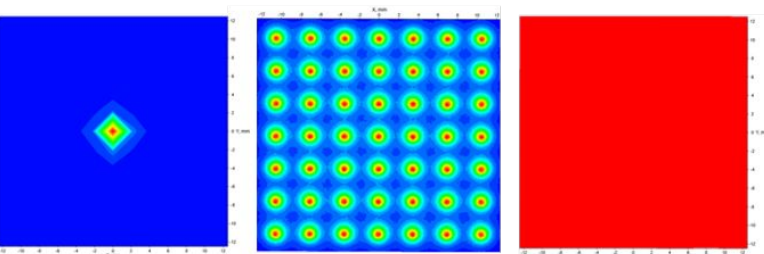

(b)
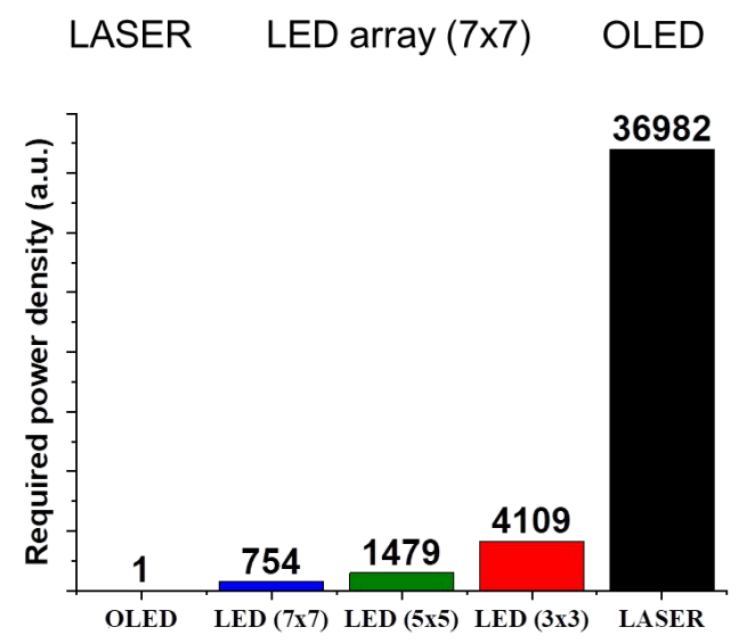

Figure S1. Comparison of optical properties according to the type of light source (a) Comparison of uniformity according to light source (LASER, LED array, OLED) (b) Comparison of power density required for a specific quantity of light (OLED emission area $=2.5 \mathrm{~cm} \times 2.5 \mathrm{~cm}$, LED and LASER emission area $=130 \mu \mathrm{m} \times 130 \mu \mathrm{m})$ 
(a)

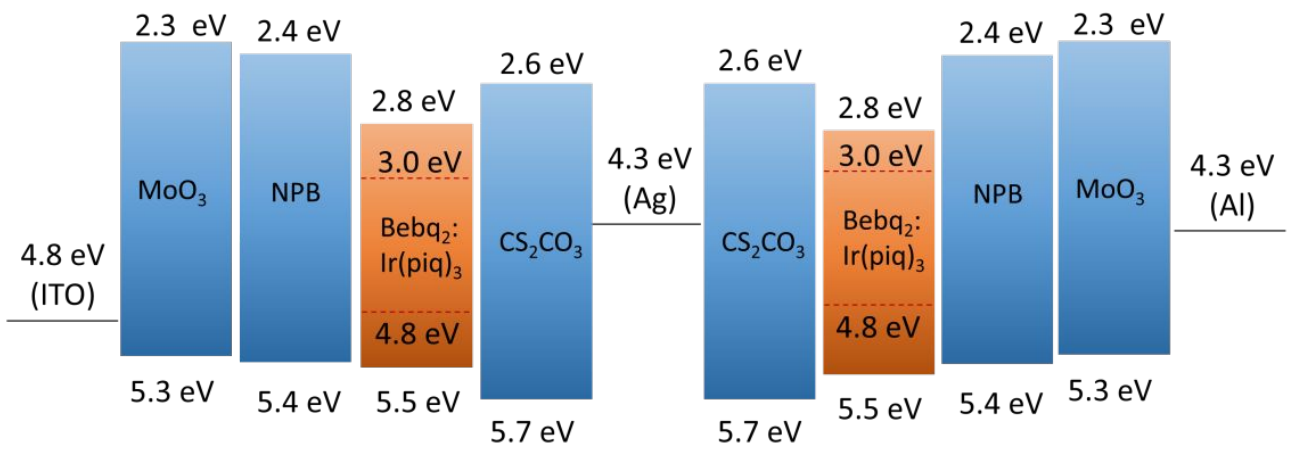

(b)

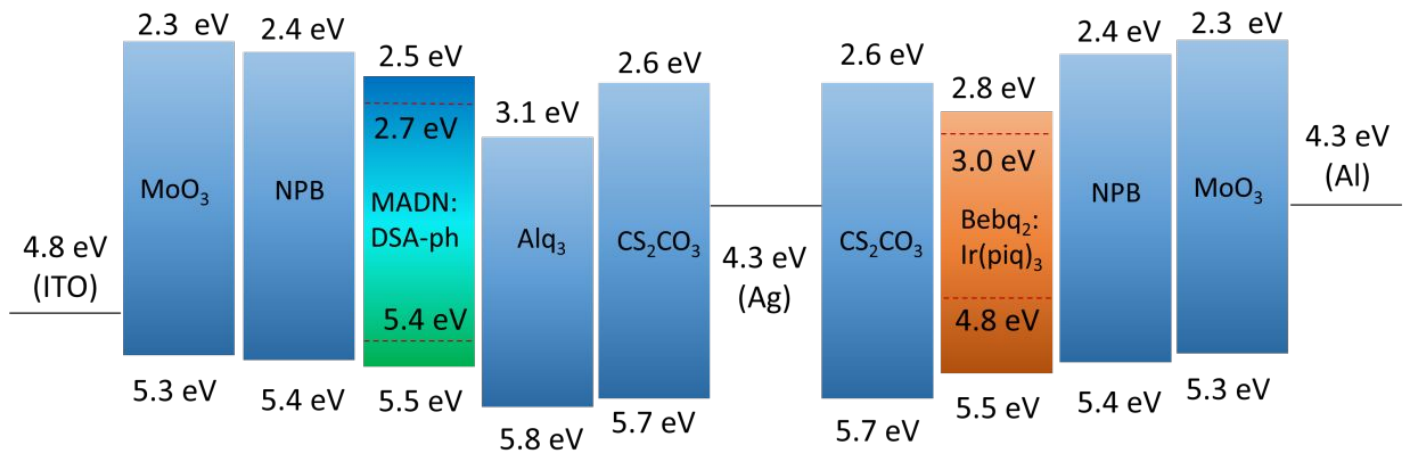

Figure S2. PAOLED structure and energy band diagram (a) The red PAOLED design is based on the energy band diagram, to control the interactions between each layer and their optoelectronic properties. (b) The energy band diagram of the white PAOLED. 


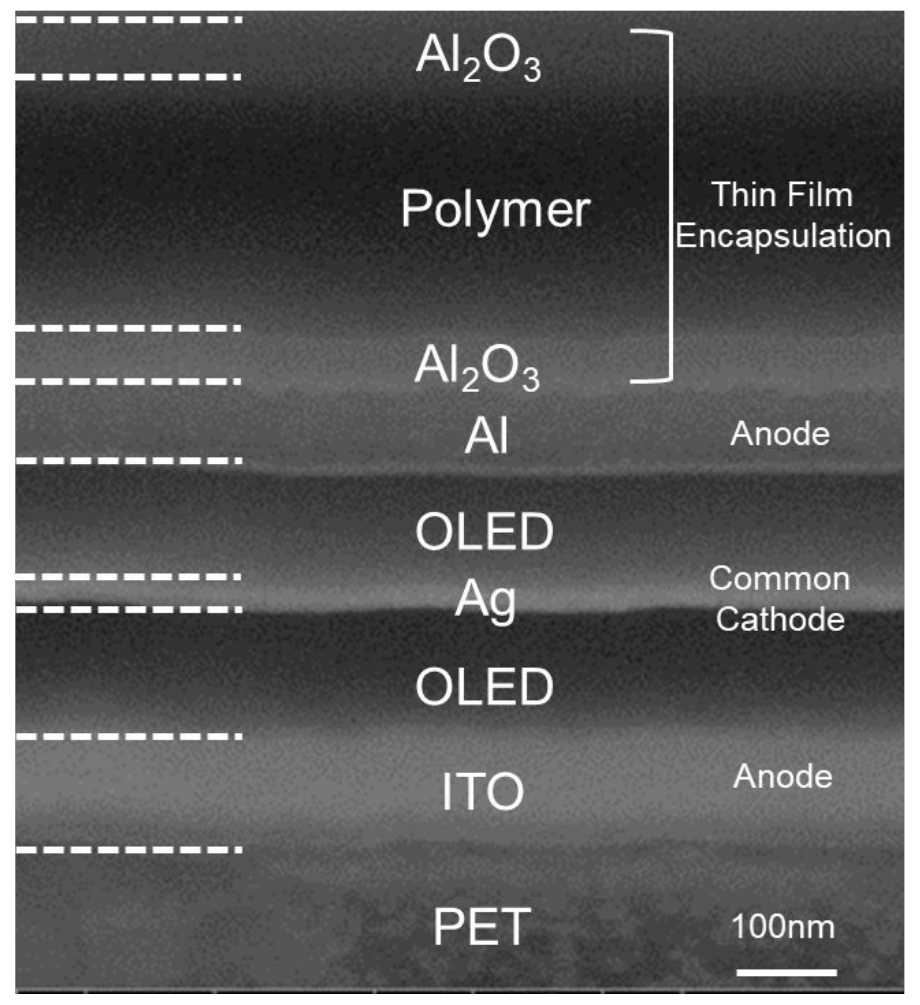

Figure S3. PAOLED's FIB-SEM cross-sectional image. 


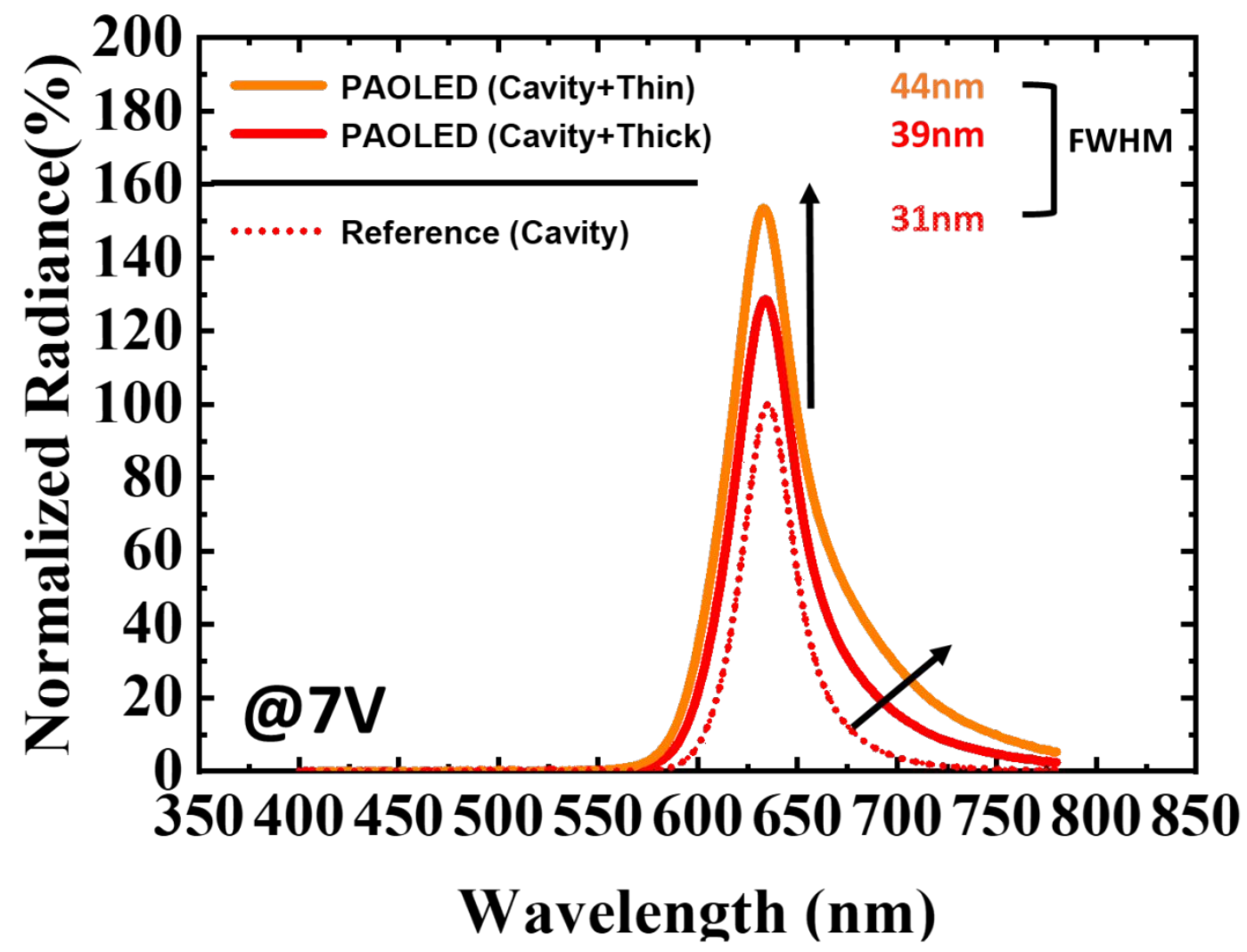

Figure S4. Graph of radiance according to the wavelength of the thin-PAOLED, thick-PAOLED compared to the reference micro-cavity OLED. 
(a)

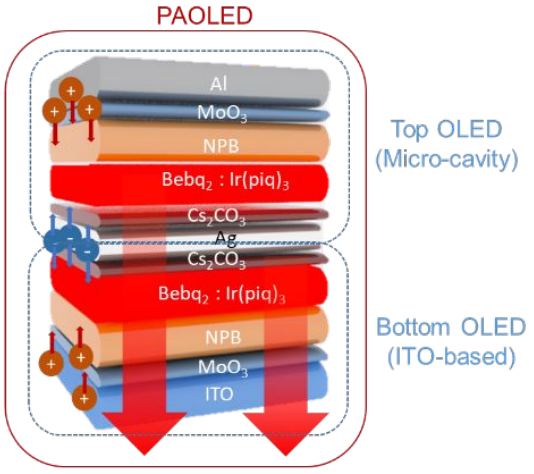

(c)

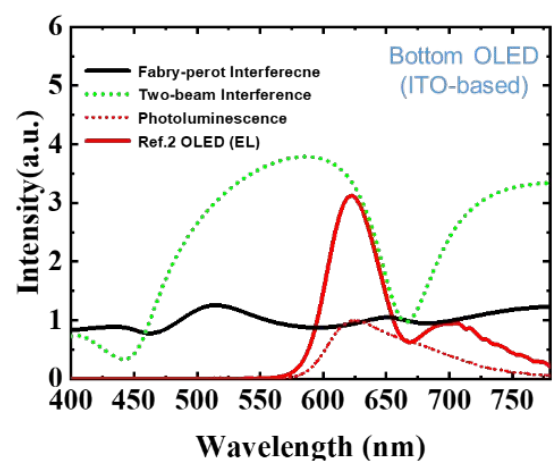

(b)

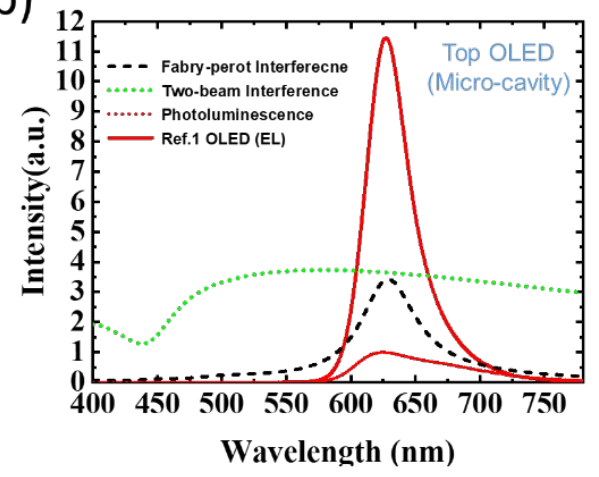

(d)

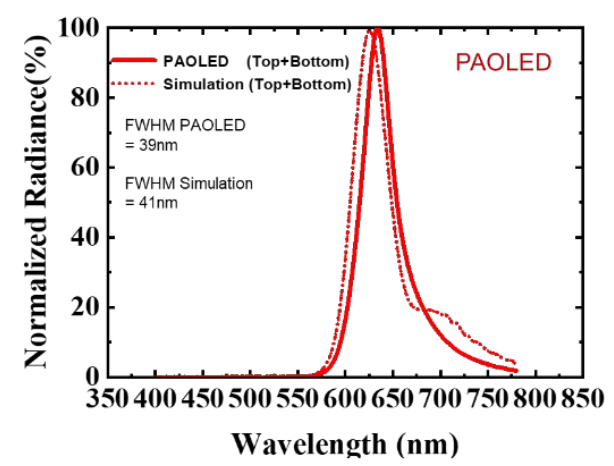

Figure S5. Wavelength analysis of the reference OLEDs and the PAOLED: (a) The structure of the PAOLED with the reference OLEDs located at the top and bottom, stacked electrically in parallel. (b) Micro-cavity OLED wavelength analysis calculated through simulation. (c) ITO-based OLED wavelength analysis calculated through simulation. (d) Comparison of measured wavelength results by manufactured PAOLED and calculated wavelength results through simulation. 


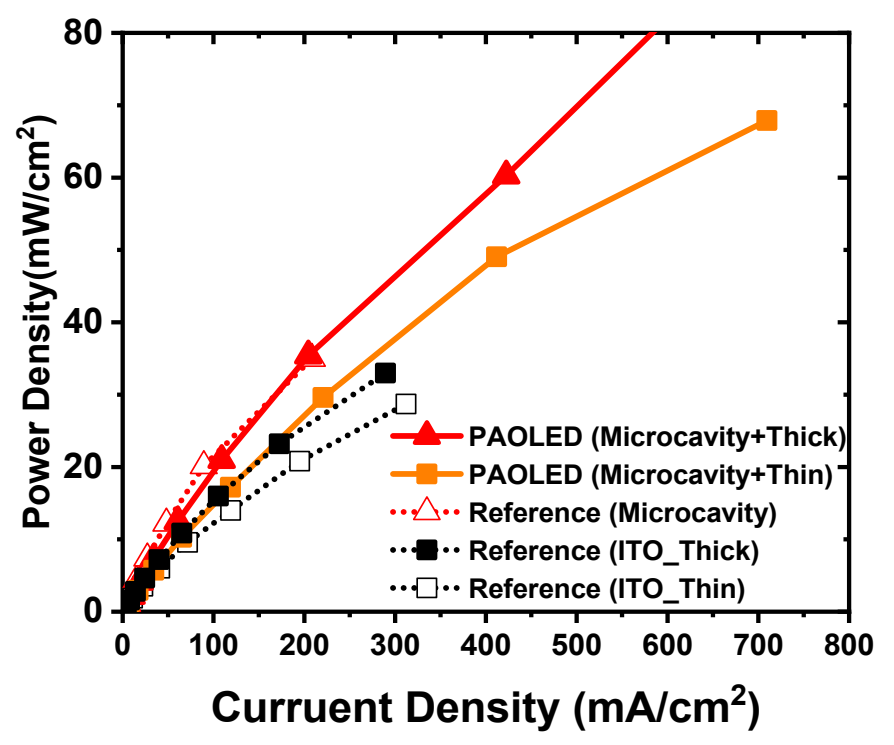

Figure S6. Power density characteristic graph according to the current density of the reference OLEDs and the PAOLED manufactured for each condition. 


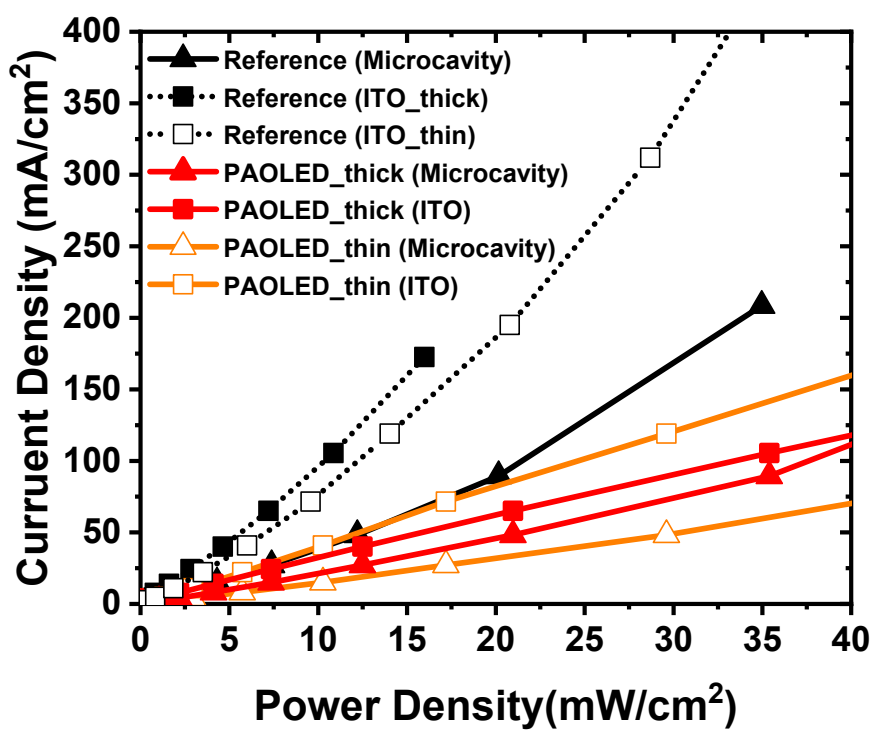

Figure S7. Required current density according to the power density of OLEDs 


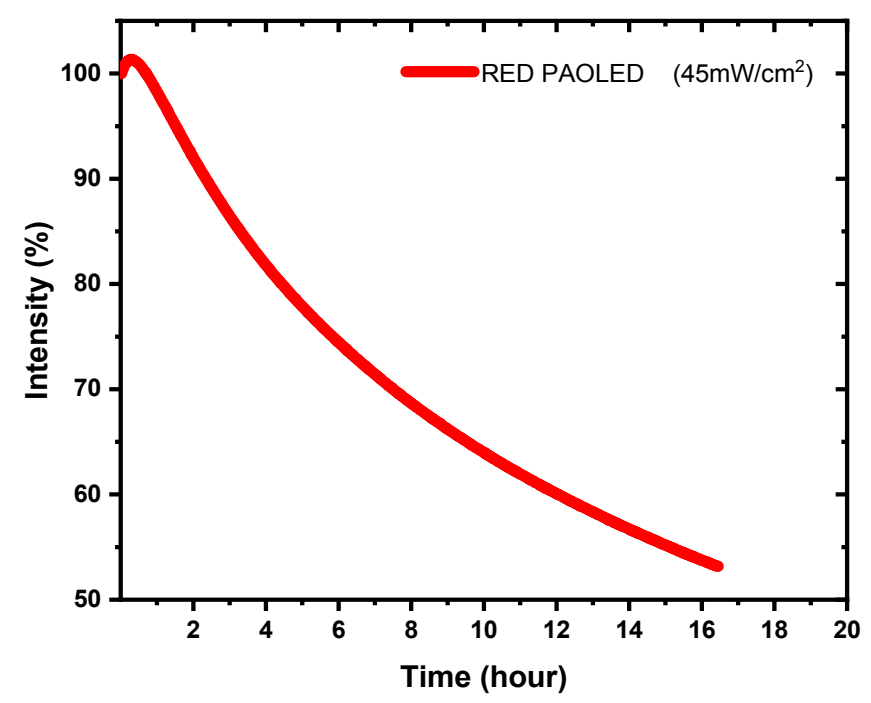

Figure S8. Operating lifetime under thick-PAOLED high-power condition $\left(45 \mathrm{~mW} / \mathrm{cm}^{2}\right)$. 


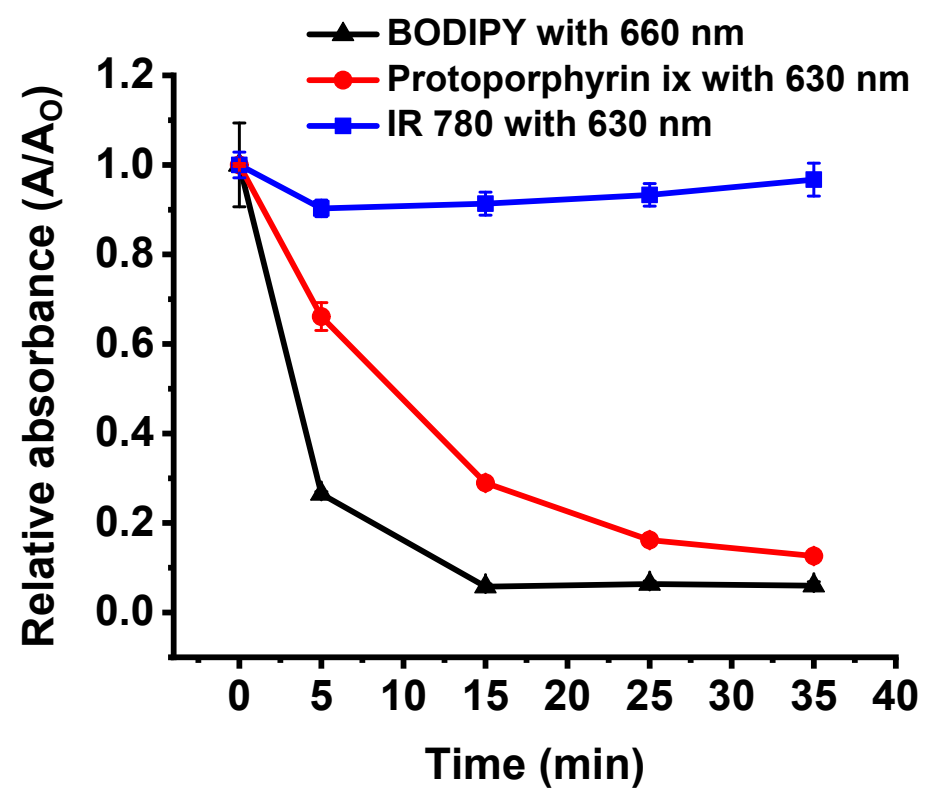

Figure S9. Singlet oxygen generation experiment using DPBF (1,3-diphenylisobenzofuran) when PAOLED $\left(35 \mathrm{~mW} / \mathrm{cm}^{2}\right)$ was irradiated, with various types of photosensitizers (BODIPY, Protoporphyrin ix, IR 780). 